\title{
On Retracts, Absolute Retracts, and Folds in COGRAPHS
}

\author{
Ton Kloks ${ }^{1}$ and Yue-Li Wang ${ }^{2}$ \\ 1 Department of Computer Science \\ National Tsing Hua University, Taiwan \\ kloks@cs.nthu.edu.tw \\ 2 Department of Information Management \\ National Taiwan University of Science and Technology \\ ylwang@cs.ntust.edu.tw
}

\begin{abstract}
Let $\mathrm{G}$ and $\mathrm{H}$ be two cographs. We show that the problem to determine whether $\mathrm{H}$ is a retract of $\mathrm{G}$ is NP-complete. We show that this problem is fixed-parameter tractable when parameterized by the size of $\mathrm{H}$. When restricted to the class of threshold graphs or to the class of trivially perfect graphs, the problem becomes tractable in polynomial time. The problem is also soluble in linear time when one cograph is given as an induced subgraph of the other. Foldings generalize retractions. We show that the problem to fold a trivially perfect graph onto a largest possible clique is NP-complete. For a threshold graph this folding number equals its chromatic number and achromatic number. We characterize the absolute retracts of cographs.
\end{abstract}

\section{Introduction}

Graph homomorphisms have regained a lot of interest by the recent characterization of Grohe of the classes of graphs for which $\operatorname{Hom}(\mathcal{G},-)$ is tractable [26]. To be precise, Grohe proves that, unless FPT $=W[1]$, deciding whether there is a homomorphism from a graph $\mathrm{G} \in \mathcal{G}$ to some arbitrary graph $\mathrm{H}$ is polynomial if and only if the graphs in $\mathcal{G}$ have bounded treewidth modulo homomorphic equivalence. The treewidth of a graph modulo homomorphic equivalence is defined as the treewidth of its core, ie, a minimal retract. This, and other recent results (see eg [4 5|13|21|28 48|49]), make it desirable to have algorithms that compute cores, or general retracts in graphs.

For any graph $\mathrm{G}$, all the cores of $\mathrm{G}$ are isomorphic subgraphs of $\mathrm{G}$. Therefore, one speaks of the core of a graph. However, a fixed copy of the core in G is not necessarily a retract. Therefore, when studying retracts or cores one usually assumes that the objective is given as an induced subgraph of $\mathrm{G}$. When restricted to cographs, when $\mathrm{H}$ is given as an induced subgraph of $\mathrm{G}$, it can be determined in linear time whether $\mathrm{H}$ is a retract. We prove this is Section 5. In the rest of the paper we do not assume that the graph $\mathrm{H}$ is given as an induced subgraph 
of G. In that case the problem turns out to be NP-complete. We prove that in Section 4

In this paper we consider the retract problem for cographs. The related surjective graph homomorphism problem was recently studied in [22]. In this paper it was shown that that the problem to decide whether there is a surjective homomorphism from one connected cograph to another connected cograph is NPcomplete. The surjective homomorphism problem is also NP-complete if both graphs are unions of complete graphs. Let us mention also the classic result of Damaschke, which is that the induced subgraph isomorphism problem is NPcomplete for cographs [12].

The retract problem for cographs can be perceived as a pattern recognition problem for labeled trees. Many pattern recognition variants have been investigated and classified, see eg, [9|12 $25 \mid 38$ |39|47|51|52|53|54]. This last manuscript [54] contains references to a lot of the work done on motifs in graphs. However, the pattern recognition problem that corresponds with the retract problem on cographs seems to have eluded all these investigations [27].

For basic terminology on graph homomorphisms we refer to [30|35].

Definition 1. Let $\mathrm{G}$ and $\mathrm{H}$ be graphs. A homomorphism $\phi: \mathrm{G} \rightarrow \mathrm{H}$ is a map $\phi: \mathrm{V}(\mathrm{G}) \rightarrow \mathrm{V}(\mathrm{H})$ which preserves edges, that is,

$$
\{x, y\} \in E(G) \quad \Rightarrow \quad\{\phi(x), \phi(y)\} \in E(H) .
$$

We write $\mathrm{G} \rightarrow \mathrm{H}$ if there is a homomorphism $\phi: \mathrm{G} \rightarrow \mathrm{H}$.

Notice that

$$
\mathrm{G} \rightarrow \mathrm{K}_{\mathrm{k}} \Leftrightarrow \chi(\mathrm{G}) \leqslant k \text { and also that } \mathrm{K}_{\mathrm{k}} \rightarrow \mathrm{G} \quad \Leftrightarrow \quad \omega(\mathrm{G}) \geqslant k .
$$

Definition 2. Let $\mathrm{G}$ and $\mathrm{H}$ be graphs. The graph $\mathrm{H}$ is a retract of $\mathrm{G}$ if there exist homomorphisms $\rho: \mathrm{G} \rightarrow \mathrm{H}$ and $\gamma: \mathrm{H} \rightarrow \mathrm{G}$ such that $\rho \circ \gamma=i \mathrm{~d}_{\mathrm{H}}$, which is the identity map $\mathrm{V}(\mathrm{H}) \rightarrow \mathrm{V}(\mathrm{H})$.

The functions $\rho$ and $\gamma$ are called the retraction and co-retraction, respectively.

When $\mathrm{H}$ is a retract of $\mathrm{G}$ then $\mathrm{H}$ is isomorphic to an induced subgraph of G. Since there are homomorphisms in two directions, $\mathrm{G}$ and $\mathrm{H}$ have the same clique number, chromatic number and odd girth. Also, there is a retraction from $G$ to $K_{k}$ if and only if $\chi(G)=\omega(G)=k$.

There is a homomorphism $\mathrm{G} \rightarrow \mathrm{H}$ if and only if the union of $\mathrm{G}$ and $\mathrm{H}$ retracts to $\mathrm{H}$. For any graph $\mathrm{H}$, checking if there is a homomorphism $\mathrm{G} \rightarrow \mathrm{H}$ is polynomial when $\mathrm{H}$ is bipartite and it is NP-complete otherwise [33]. It follows that, 
for any graph $\mathrm{H}$, checking if a graph $\mathrm{H}$ is a retract of a graph $\mathrm{G}$ is NP-complete, unless $\mathrm{H}$ is bipartite. The problem remains NP-complete, even when $\mathrm{H}$ is an even cycle of length at least six, given as an induced subgraph of $\mathrm{G}$ [16]. The question whether a graph $\mathrm{G}$ has a homomorphism to itself which is not the identity is also NP-complete [34].

Definition 3. A graph is a cograph if it has no induced $\mathrm{P}_{4}$, which is the path with four vertices.

Since the complement of a $\mathrm{P}_{4}$ is a $\mathrm{P}_{4}$, cographs are closed under complementation. Actually, the class of cographs is the smallest class of graphs which is closed under complementation and taking unions.

A similar characterization of cographs reads as follows. A graph $\mathrm{G}$ is a cograph if and only if one of the following holds.

(1) G has only one vertex, or

(2) $G$ is disconnected and every component is a cograph, or

(3) the complement of $G, \bar{G}$ is disconnected and every component of $\bar{G}$ is a cograph.

It follows that cographs have a decomposition tree, called a cotree, defined as follows. The decomposition tree is a rooted tree $T$. There is a bijection from the leaves of $T$ to the vertices of $G$. When $G$ has at least two vertices then each internal node of T, including the root, is labeled as $\otimes$ or $\oplus$. The $\oplus$ label at a node takes the union of the graphs that correspond with the children of the node. The $\otimes$ label takes the join of the graphs that correspond with the children.

Remark 1. When defined as above, the labels of the internal nodes in any path from the root to a leaf alternate between $\oplus$ and $\otimes$. Alternatively, one frequently defines a cotree as a rooted binary tree, in which each internal node is labeled as $\oplus$ and $\otimes$. In this paper, when talking about cotrees, we always assume the first type of cotree. Thus, each child of the root corresponds with one component or, with one cocomponent of the graph.

Remark 2. It is well-known that cographs are recognizable in linear time [11|20]. A cotree has $\mathrm{O}(\mathrm{n})$ nodes, where $\mathrm{n}=|\mathrm{V}(\mathrm{G})|$, and it can be obtained in linear time.

This paper is organized as follows. In Sections 2 and 3 we show that the retract problem is polynomial when restricted to the classes of threshold and trivially perfect graphs. In Section 4 we show that the problem is NP-complete for cographs. In Section 5 we show that, when $\mathrm{H}$ is given as an induced subgraph of $G$, it can be determined in polynomial time whether $H$ is a retract of G. In Section 6 we show that the retract problem for cographs is fixedparameter tractable. In Section 7 we show that computing the folding number is NP-complete for trivially perfect graphs. For threshold graphs the folding number equals the chromatic and achromatic number. In Section 9 we mention some of our ideas for future research. 


\section{Retracts in threshold graphs}

A subclass of the class of cographs is the class of threshold graphs. Threshold graphs are the graphs without induced $2 \mathrm{~K}_{2}, \mathrm{C}_{4}$ and $\mathrm{P}_{4}$. We use the following characterization of threshold graphs.

Theorem 1. A graph is a threshold graph if and only if every induced subgraph has a universal vertex or an isolated vertex.

Theorem 2. Let $\mathrm{G}$ and $\mathrm{H}$ be threshold graphs. There exists a linear-time algorithm to check if $\mathrm{H}$ is a retract of $\mathrm{G}$.

Proof. Assume that $\mathrm{H}$ is a retract of $\mathrm{G}$ and let $\rho$ and $\gamma$ be the retraction and co-retraction.

Assume that $G$ has a universal vertex, say $x_{1}$. Then $H$ must have a universal vertex as well, since a retract of a connected graph is connected. Let $y_{1}$ be a universal vertex of $H$. Let $y_{i}=\rho\left(x_{1}\right)$. Since $\rho$ is a homomorphism it preserves edges, and since $x_{1}$ is universal in $G, \rho$ maps no other vertex of $G$ to $y_{i}$. Notice also that $\gamma\left(y_{i}\right)=x_{1}$ since $\rho \circ \gamma=i d_{H}$ and $\rho$ maps no other vertex to $y_{i}$.

Assume that $y_{i} \neq y_{1}$. Let $\gamma\left(y_{1}\right)=x_{\ell}$. Then $x_{\ell} \neq x_{1}$ since $\gamma$ preserves edges and so

$$
\left\{y_{1}, y_{i}\right\} \in E(H) \Rightarrow\left\{\gamma\left(y_{1}\right), \gamma\left(y_{i}\right)\right\}=\left\{x_{\ell}, x_{1}\right\} \in E(G) \quad \Rightarrow \quad x_{\ell} \neq x_{1} .
$$

Furthermore, since $y_{1}$ is universal, $\gamma$ maps no other vertex of $H$ to $x_{\ell}$. Of course, since $\rho \circ \gamma=i_{H}, \rho\left(x_{\ell}\right)=y_{1}$.

We claim that $y_{i}$ is universal in $H$, and therefore exchangeable with $y_{1}$. Assume not and let $y_{s} \in V(H)$ be another vertex of $H$ not adjacent to $y_{i}$. Let $\gamma\left(y_{s}\right)=x_{p}$. Then $x_{p} \neq x_{1}$ since $\rho \circ \gamma=i d_{H}$ and $\rho\left(x_{1}\right)=y_{i} \neq y_{s}$. Now, since $\rho$ is a homomorphism,

$$
\left\{x_{1}, x_{p}\right\} \in E(G) \Rightarrow\left\{\rho\left(x_{1}\right), \rho\left(x_{p}\right)\right\}=\left\{y_{i}, y_{s}\right\} \in E(H),
$$

which is a contradiction. Therefore, we may assume that $y_{i}=y_{1}$.

$$
\begin{gathered}
\text { That is, from now on we assume that } \\
\rho\left(x_{1}\right)=y_{1} \text { and } \gamma\left(y_{1}\right)=x_{1} .
\end{gathered}
$$

This proves that, when $G$ is connected then $H$ is a retract of $G$ if and only if $H-y_{1}$ is a retract of $G-x_{1}$. By the way, notice that if $|V(H)|=1$ then $H$ can be a retract of $\mathrm{G}$ only if $\mathrm{G}$ is an independent set, so this case is easy to check.

Finally, assume that $\mathrm{G}$ is not connected. Since $\mathrm{G}$ has no induced $2 \mathrm{~K}_{2}$, all components, except possibly one, have only one vertex. The number of components of $\mathrm{H}$ can be at most equal to the number of components of $\mathrm{G}$, since $\rho$ maps components in $\mathrm{G}$ to components of $\mathrm{H}$, and $\rho \circ \gamma=i d_{\mathrm{H}}$, and so any two components of $\mathrm{H}$ are mapped by $\gamma$ to different components of $\mathrm{G}$. 
First assume that $\mathrm{H}$ is also disconnected. Let $x_{1}, \ldots, x_{a}$ be the isolated vertices of $G$ and let $y_{1}, \ldots, y_{b}$ be the isolated vertices of $H$. Let $\rho\left(x_{i}\right)=y_{i}$ and $\gamma\left(y_{i}\right)=x_{i}$ for $i \in\{1, \ldots, b\}$ and let $\rho\left(x_{b+1}\right)=\cdots=\rho\left(x_{a}\right)=y_{b}$. Now, $H$ is a retract of $G$ if and only if $H-\left\{x_{1}, \ldots, x_{b}\right\}$ is a retract of $G-\left\{x_{1}, \ldots, x_{a}\right\}$.

If $H$ is connected, with at least two vertices, then let $y_{1}$ be a universal vertex and let $\rho\left(x_{1}\right)=\cdots=\rho\left(x_{a}\right)=y_{1}$. If $H$ is a retract of $G$ then $G$ must have exactly one component with at least two vertices, since $G$ is a threshold graph and $\rho$ is a homomorphism. Let $x_{\mathfrak{u}}$ be the universal vertex of that component and define $\rho\left(x_{\mathfrak{u}}\right)=y_{1}$ and $\gamma\left(y_{1}\right)=x_{\mathfrak{u}}$. In this case, $H$ is a retract if and only if $H-y_{1}$ is a retract of $G-\left\{x_{1}, \ldots, x_{a}, x_{u}\right\}$.

An elimination ordering, which eliminates successive isolated and universal vertices in a threshold graph, can be obtained in linear time. This proves the theorem.

\section{Retracts in trivially perfect graphs}

Definition 4 ([2358]). A graph $\mathrm{G}$ is trivially perfect if for all induced subgraphs $\mathrm{H}$ of $\mathrm{G}, \alpha(\mathrm{H})$ is equal to the number of maximal cliques in $\mathrm{H}$.

Trivially perfect graphs are those graphs without induced $\mathrm{C}_{4}$ and $\mathrm{P}_{4}$.

Theorem 3 ([58]). A graph is trivially perfect if and only if every connected induced subgraph has a universal vertex.

Theorem 4. Let $\mathrm{G}$ and $\mathrm{H}$ be trivially perfect graphs. There exists an $\mathrm{O}\left(\mathrm{N}^{5 / 2}\right)$ algorithm which checks if $\mathrm{H}$ is a retract of $\mathrm{G}$, where $\mathrm{N}=|\mathrm{V}(\mathrm{G})| \cdot|\mathrm{V}(\mathrm{H})|$.

Proof. Assume that $\mathrm{H}$ is a retract of $\mathrm{G}$. Let $\mathrm{C}_{1}, \ldots, \mathrm{C}_{\mathrm{t}}$ be the components of $G$ and let $D_{1}, \ldots, D_{s}$ be the components of $H$. Then $s \leqslant t$. Without loss of generality, let $D_{i}$ be a retract of $C_{i}$ for $i \in\{1, \ldots, s\}$. For the components $C_{i}$ with $i>s$, there must be a $j \leqslant s$ such that there is a homomorphism from $C_{i}$ to $D_{j}$.

First assume that $G$ and $H$ are connected. Let $g_{1}, \ldots, g_{k}$ be the universal vertices of $G$ and let $h_{1}, \ldots, h_{\ell}$ be the universal vertices of $H$. As in the proof of Theorem 2 it follows that $\mathrm{H}$ is a retract of $\mathrm{G}$ if and only if

(i) $\ell \geqslant k$, and

(ii) either $\mathrm{H}$ is a clique and $\omega(\mathrm{G})=\omega(\mathrm{H})$ or $\mathrm{H}-\left\{\mathrm{h}_{1}, \ldots, \mathrm{h}_{\mathrm{k}}\right\}$ is a retract of $\mathrm{G}-\left\{\mathrm{g}_{1}, \ldots, \mathrm{g}_{\mathrm{k}}\right\}$.

For the general case, consider the following bipartite graph $\mathrm{B}$. The vertices of $\mathrm{B}$ are the components of $G$ and $H$. There is an edge $\left\{C_{i}, D_{j}\right\} \in E(B)$ if and only if $C_{i}$ retracts to $D_{j}$. Then $G$ retracts to $H$ if and only if

(a) B has a matching which exhausts all components of $\mathrm{H}$, and

(b) for every component $C_{i}$ which is not an endpoint of an edge in the matching there is a $D_{j}$ such that there is a homomorphism from $G\left[C_{i}\right]$ to $H\left[D_{j}\right]$. 
To check if a component $G\left[C_{i}\right]$ retracts to some $H\left[D_{j}\right]$ the algorithm greedily matches the universal vertices of $G\left[C_{i}\right]$ and $H\left[D_{j}\right]$ and checks if the remaining graph $\mathrm{G}^{\prime}$, ie, after removal of the matched universal vertices, retracts to the remaining graph $\mathrm{H}^{\prime}$. Let $C_{i}^{1}, \ldots, C_{i}^{p}$ and $D_{j}^{1}, \ldots, D_{j}^{q}$ be the components of $G^{\prime}$ and $H^{\prime}$. The algorithm constructs the bipartite graph $B_{i j}$ on the components $C_{i}^{k}$ and $D_{j}^{\ell}$, where $k \in\{1, \ldots, p\}$ and $\ell \in\{1, \ldots, q\}$. The algorithm checks if there is an edge $\left(C_{i}^{k}, D_{j}^{\ell}\right) \in E\left(B_{i j}\right)$ in $O(1)$ time by table look-up, and so the bipartite graph $B_{i j}$ is constructed in

$$
\mathrm{O}(\mathrm{pq})=\mathrm{O}\left(\left|\mathrm{C}_{\mathrm{i}}\right| \cdot\left|\mathrm{D}_{j}\right|\right) .
$$

Edmonds' algorithm [14] computes a maximum matching in $B_{i j}$ in time

$$
\mathrm{O}\left((p+q)^{5 / 2}\right)=\mathrm{O}\left(\left(\left|C_{i}\right|+\left|D_{j}\right|\right)^{5 / 2}\right) .
$$

Summing over the components $C_{i}$ and $D_{j}$, for $i \in\{1, \ldots, t\}$ and $j \in\{1, \ldots, s\}$, we obtain

$$
\sum_{i=1}^{t} \sum_{j=1}^{s}\left|C_{i}\right| \cdot\left|D_{j}\right|+\left(\left|C_{i}\right|+\left|D_{j}\right|\right)^{5 / 2}=O\left(|V(G)|^{5 / 2} \cdot|V(H)|^{5 / 2}\right) .
$$

This proves the claim.

\section{NP-completeness of retracts in cographs}

Recall that a graph $G$ is perfect when $\omega\left(\mathrm{G}^{\prime}\right)=\chi\left(\mathrm{G}^{\prime}\right)$ for every induced subgraph $G^{\prime}$ of $G$. By the perfect graph theorem a graph is perfect if and only if it has no odd hole or odd antihole [8]. This implies that cographs are perfect. Perfect graphs are recognizable in polynomial time [7]. For a graph G, when $\omega(\mathrm{G})=\chi(\mathrm{G})$ one can compute this value in polynomial time via Lovász theta function [29].

The following lemma appears, eg, in [18].

Lemma 1. Assume that $\omega(\mathrm{H})=\chi(\mathrm{H})$. There is a homomorphism $\mathrm{G} \rightarrow \mathrm{H}$ if and only if $\chi(\mathrm{G}) \leqslant \omega(\mathrm{H})$.

Proof. Write $\omega=\omega(H)=\chi(H)$. First assume that there is a homomorphism $\phi: G \rightarrow H$. There is a homomorphism $\mathrm{f}: \mathrm{H} \rightarrow \mathrm{K}_{\omega}$ since $\mathrm{H}$ is $\omega$-colorable. Then $f \circ \phi: G \rightarrow K_{\omega}$ is a homomorphism, and so $G$ has a $\omega$-coloring. This implies that $\chi(G) \leqslant \omega$.

Assume $\chi(G) \leqslant \omega$. There is a homomorphism $G \rightarrow K_{k}$, where $k=\chi(G)$. Since $\mathrm{K}_{\mathrm{k}}$ is an induced subgraph of $\mathrm{H}$, there is also a homomorphism $\mathrm{K}_{\mathrm{k}} \rightarrow \mathrm{H}$. This implies that $\mathrm{G}$ is homomorphic to $\mathrm{H}$, ie $\mathrm{G} \rightarrow \mathrm{H}$.

Corollary 1. When $\mathrm{G}$ and $\mathrm{H}$ are perfect one can check in polynomial time whether there is a homomorphism $\mathrm{G} \rightarrow \mathrm{H}$. 
It is well-known that retracts, like general homomorphisms, constitute a transitive relation. We provide a short proof for completeness sake.

Lemma 2. Let $\mathrm{A}$ be a retract of $\mathrm{G}$ and let $\mathrm{B}$ be a retract of $\mathrm{A}$. Then $\mathrm{B}$ is a retract of $\mathrm{G}$.

Proof. Let $\rho_{1}$ and $\gamma_{1}$ be a retraction and co-retraction from $G$ to $A$ and let $\rho_{2}$ and $\gamma_{2}$ be a retraction and co-retraction from $A$ to B. Since all four maps $\rho_{1}, \rho_{2}$, $\gamma_{1}$ and $\gamma_{2}$ are homomorphisms, the following two maps are homomorphisms as well.

$$
\rho_{2} \circ \rho_{1}: G \rightarrow B \text { and } \gamma_{1} \circ \gamma_{2}: B \rightarrow G \text {. }
$$

Furthermore,

$$
\left(\rho_{2} \circ \rho_{1}\right) \circ\left(\gamma_{1} \circ \gamma_{2}\right)=\rho_{2} \circ i d_{A} \circ \gamma_{2}=\rho_{2} \circ \gamma_{2}=i d_{B} .
$$

This proves that $B$ is a retract of $G$.

Throughout the remainder of this section it is assumed that $\mathrm{G}$ and $\mathrm{H}$ are cographs. Note that, using the cotree, $\omega(\mathrm{G})$ and $\chi(\mathrm{G})$ can be computed in linear time when $\mathrm{G}$ is a cograph.

Lemma 3. Assume $\mathrm{H}$ is disconnected, with components $\mathrm{H}_{1}, \ldots, \mathrm{H}_{\mathrm{t}}$. Assume that $\mathrm{H}$ is a retract of a graph $\mathrm{G}$. Then there is an ordering of the components of $\mathrm{G}$, say $\mathrm{G}_{1}, \ldots, \mathrm{G}_{\mathrm{s}}$ such that

(a) $s \geqslant t$, and

(b) $G_{i}$ retracts to $H_{i}$, for every $i \in\{1, \ldots, t\}$, and

(c) for every $j \in\{t+1, \ldots, s\}$, there is a homomorphism $\mathrm{G}_{j} \rightarrow \mathrm{H}$.

Proof. No connected graph has a disconnected retract since the homomorphic image of a connected graph is connected. To see that, notice that a homomorphism $\phi: G \rightarrow H$ is a vertex coloring of $G$, where the vertices of $H$ represent colors. By that we mean that, for each $v \in \mathrm{V}(\mathrm{H})$, the pre-image $\phi^{-1}(v)$ is an independent set in $G$ or $\varnothing$. One obtains the image $\phi(G)$ by identifying vertices in $\mathrm{G}$ that receive the same color. When $\mathrm{G}$ is connected, this 'quotient graph' on the color classes is also connected, which is easy to prove by means of contradiction.

Assume that $G$ retracts to $H$. Then we may assume that $H_{1}, \ldots, H_{t}$ are induced subgraphs of components $G_{1}, \ldots, G_{t}$ of $G$ and that each $G_{i}$ retracts to $H_{i}$. For the remaining components $G_{j}$, where $j>t$, there is then a homomorphisms $\mathrm{G}_{j} \rightarrow \mathrm{H}$.

Notice that, for $j>t$, we can check if there is a homomorphism $G_{j} \rightarrow H$ by checking if $G_{j} \oplus H_{k}$ retracts to $H_{k}$, for some $1 \leqslant k \leqslant t$ (see, eg, [56]), or, equivalently (since cographs are perfect), if $\omega\left(G_{j}\right) \leqslant \omega\left(H_{k}\right)$ for some $1 \leqslant k \leqslant t$. 
Remark 3. Assume that we are given, for each pair $G_{i}$ and $H_{j}$ whether $G_{i}$ retracts to $\mathrm{H}_{j}$ or not. Then, to check if $\mathrm{G}$ retracts to $\mathrm{H}$, we may consider a bipartite graph B defined as follows (see eg [952]). One color class of B has the components of $\mathrm{G}$ as vertices and the other color class has the components of $\mathrm{H}$ as vertices. There is an edge between $G_{i}$ and $H_{j}$ whenever $G_{i}$ retracts to $H_{j}$. To check if $\mathrm{G}$ retracts to $\mathrm{H}$, we can let an algorithm compute a maximum matching in $\mathrm{B}$. There is a retraction only if the matching exhausts all components of $\mathrm{H}$ and if $\omega(\mathrm{G})=\omega(\mathrm{H})$.

A cocomponent of a graph $\mathrm{G}$ is a subset of vertices which induces a component of the complement $\bar{G}$.

Lemma 4. Assume $\mathrm{G}$ is connected and assume that $\mathrm{G}$ retracts to $\mathrm{H}$. Then $\mathrm{H}$ is also connected. Let $\mathrm{G}_{1}, \ldots, \mathrm{G}_{\mathrm{t}}$ be the subgraphs of $\mathrm{G}$ induced by the cocomponents of $\mathrm{G}$. Then there is a partition of the cocomponents of $\mathrm{H}$ such that the subgraphs of $\mathrm{H}$ induced by the parts of the partition, can be ordered $\mathrm{H}_{1}, \ldots, \mathrm{H}_{\mathrm{t}}$ such that $\mathrm{G}_{i}$ retracts to $\mathrm{H}_{i}$ for $i \in\{1, \ldots, t\}$.

Proof. Every subgraph $G_{i}$ of $G$, induced by a cocomponent, retracts to some induced subgraph. These retracts are pairwise joined, so each part is the join of some subgraphs induced by cocomponents of $\mathrm{H}$. Thus the parts of $\mathrm{V}(\mathrm{H})$ that are the images of the subgraphs induced by cocomponents of $G$ form a partition of the cocomponents of $\mathrm{H}$.

Theorem 5. Let $\mathrm{G}$ and $\mathrm{H}$ be cographs. The problem to decide whether $\mathrm{H}$ is a retract of $\mathrm{G}$ is NP-complete.

Proof. We reduce the 3-partition problem to the retract problem on cotrees. The 3-partition problem is the following. Let $\mathrm{m}$ and B be integers. Let $\mathrm{S}$ be a multiset of $3 \mathrm{~m}$ positive integers, $a_{1}, \ldots, a_{3 m}$. Determine if there is a partition of $S$ into $m$ subsets $S_{1}, \ldots, S_{m}$, such that the sum of the numbers in each subset is B. Without loss of generality we assume that each number is strictly between B/4 and $\mathrm{B} / 2$, which guarantees that in a solution each subset contains exactly three numbers that add up to $B$.

The 3-partition problem is strongly NP-complete, that is, the problem remains NP-complete when all the numbers in the input are represented in unary [19].

In our reduction, the cotree for the graph $\mathrm{H}$ has a root which is labeled as a join-node $\otimes$. The root has $3 m$ children, one for each number $a_{i}$. For simplicity we refer to the children as $a_{i}, i \in\{1, \ldots, 3 m\}$. Each child $a_{i}$ has a union node $\oplus$ as the root. The root of each $a_{i}$-child has two children, one is a single leaf and the other is a join-node $\otimes$ with $a_{i}$ leaves. This ends the description of $H$.

The cotree for the graph $\mathrm{G}$ has a join-node $\otimes$ as a root and this has $m$ children. The idea is that each child corresponds with one set of a 3-partition of S. The 


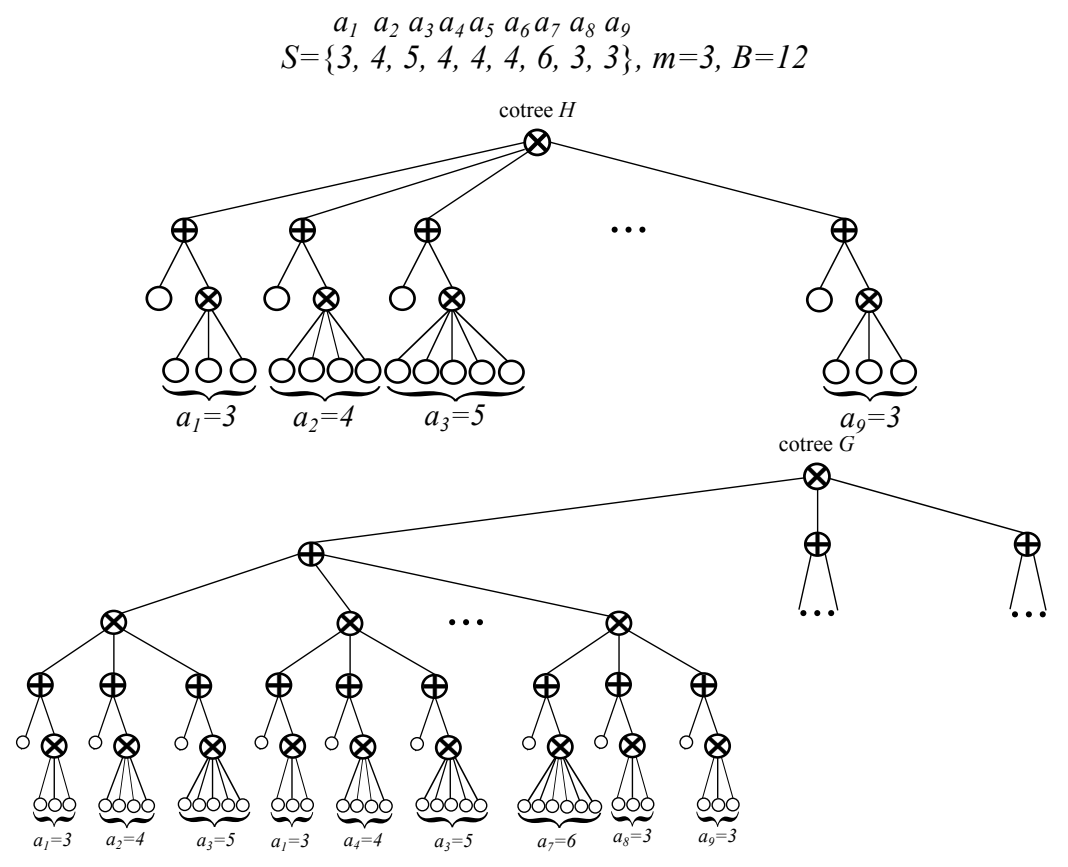

Fig. 1. The cotrees for $\mathrm{G}$ and $\mathrm{H}$ used in the proof of Theorem 5

subtrees for all the children are identical. It has a union-node $\oplus$ as the root. Consider all triples $\{i, j, k\}$ for which $a_{i}+a_{j}+a_{k}=B$. For each such triple create one child, which is the join of three cotrees, one for $a_{i}$, one for $a_{j}$ and one for $a_{k}$ in the triple. The subtree for $a_{i}$ is a union of two subtrees. As in the cotree for the pattern $\mathrm{H}$, one subtree is a single leaf, and the other subtree is the join of $a_{i}$ leaves. The other two subtrees, for the numbers $a_{j}$ and $a_{k}$ in the triple are similar.

Let $T_{H}$ and $T_{G}$ be the cotrees for $H$ and $G$ as constructed above. Say $T_{H}$ and $T_{G}$ have roots $r_{H}$ and $r_{G}$. When the graph $H$ is a retract of $G$ then the $a_{i}$-children of $r_{H}$ are partitioned into triples, such there is a bijection between these triples, say $\left\{a_{i}, a_{j}, a_{k}\right\}$ and a branch in the cotree of G. Each $\oplus$-node which is the root of a child of $r_{G}$ must have exactly one $\left\{a_{i}, a_{j}, a_{k}\right\}$-child that corresponds with the triple. Notice that, by the construction, all subgraphs induced by remaining components of the $\oplus$-node have maximal cliques of size B. Therefore, all other children of the $\oplus$-node are homomorphic to the one child which corresponds to the triple $\left\{a_{i}, a_{j}, a_{k}\right\}$.

It now follows from Lemma 4 that there is a 3-partition if and only if the graph $\mathrm{H}$ is a retract of $\mathrm{G}$. This completes the proof. 


\section{The partitioned case for retracts in cographs}

This section is dedicated to a special case, the 'partitioned case.' It deals with the subproblem where $\mathrm{H}$ is given as an induced subgraph of $\mathrm{G}$.

Theorem 6. Let $\mathrm{G}$ and $\mathrm{H}$ be cographs and assume that $\mathrm{H}$ is given as an induced subgraph of $\mathrm{G}$. By that we mean that we can test in $\mathrm{O}(1)$ time whether a given vertex of $\mathrm{G}$ is in $\mathrm{H}$ or not. There exists a linear-time algorithm to test if $\mathrm{G}$ retracts to $\mathrm{H}$.

Proof. We describe the algorithm. Construct a cotree for the graph G. Repeatedly, remove children of $\oplus$-nodes for which

(a) the branch has no leaves corresponding with vertices in $\mathrm{H}$, and

(b) the subgraph induced by the branch has clique number at most the clique number of a sibling.

When the algorithm ends such that all remaining vertices are in $\mathrm{H}$ then $\mathrm{G}$ retracts to $\mathrm{H}$ and otherwise it does not.

It is easy to check the correctness of this algorithm. Also, it is not difficult to see that it can be implemented to run in linear time in the size of G.

\section{A fixed-parameter solution for retracts in cographs}

In this section we look at a parameterized solution for the retract problem. Let $G$ and $\mathrm{H}$ be cographs. We consider the parameterization by the number of vertices in $\mathrm{H}$. Let

$$
k=|V(H)| \text {. }
$$

Lemma 5. When $\mathrm{H}$ is a retract of $\mathrm{G}$ then $\omega(\mathrm{G})=\omega(\mathrm{H}) \leqslant \mathrm{k}$. Let $\mathrm{T}_{\mathrm{G}}$ be a cotree for $\mathrm{G}$. Then every join-node in $\mathrm{T}_{\mathrm{G}}$ has at most $\mathrm{k}$ children, and the height of the cotree is $\mathrm{O}(\mathrm{k})$.

Proof. Let $\mathrm{H}$ be a retract of $\mathrm{G}$. By definition of a retract $\omega(\mathrm{G})=\omega(\mathrm{H}) \leqslant \mathrm{k}$.

Let $T_{G}$ be a cotree. Every $\otimes$-node $p$ in $T_{G}$ has at most $k$ children, since every child adds at least one vertex to a clique in the subgraph represented by $p$.

The labels on the internal nodes on a path from the root to the leaves alternate between $\oplus$ and $\otimes$. Therefore, if the height would be more than $2 k+1$ then the path contains more than $k$ join nodes. Each of these join nodes adds at least one to a clique in $\mathrm{G}$, which is a contradiction.

Theorem 7. The retract problem, which asks if a cograph $\mathrm{H}$ is a retract of $\mathrm{G}$, is fixed-parameter tractable when parameterized by the number of vertices in $\mathrm{H}$. 
Proof. Consider cotrees $T_{G}$ and $T_{H}$ for $G$ and $H$ and let $r_{G}$ and $r_{H}$ be the roots of the two cotrees. Assume both roots are $\otimes$-nodes. Then both have at most $k$ children. According to Lemma 4, when $\mathrm{H}$ is a retract of $\mathrm{G}$ there is a partition $\mathcal{P}$ of the lines incident with $r_{H}$ such that each child of $r_{G}$ represents a graph that retracts to the subgraph of $\mathrm{H}$ induced by exactly one part of the partition. Let $p$ be the number of children of $r_{G}$ and let $q$ be the number of children of $r_{H}$. The number of partitions of a $q$-set into $p$ nonempty parts is given by the Stirling number of the second kind. A trivial upperbound for the number of different assignments of the children of $r_{H}$ to the children of $r_{G}$ is

$$
p^{q} \leqslant k^{k}
$$

since, by Lemma 5 , $p \leqslant k$ and $q \leqslant k$.

Our algorithm tries all possible partitions of the children of $r_{H}$. Consider a partition $\mathcal{P}$, and let $P_{i} \in \mathcal{P}$ be mapped to the $i^{\text {th }}$ child of $r_{G}$. Let $H_{i}$ be the subgraph of $H$ induced by $P_{i}$ and let $G_{i}$ be the cocomponent of $G$ induced by the $i^{\text {th }}$ child of $r_{G}$. We proceed as in the proof of Theorem 4 , Let $C_{i}^{1}, \ldots, C_{i}^{a}$ be the components of the root of the $i^{\text {th }}$ child of $r_{G}$. Let $D_{i}^{1}, \ldots, D_{i}^{b}$ be the components of $H_{i}$. Consider the bipartite graph with vertices the components of $G_{i}$ and $H_{i}$, where an edge $\left(C_{i}^{\alpha}, D_{i}^{\beta}\right)$ indicates that $C_{i}^{\alpha}$ retracts to $D_{i}^{\beta}$. The algorithm checks if there is a matching that exhausts all components of $\mathrm{H}_{i}$, and it checks if the remaining components of $G_{i}$ are homomorphic to some component of $H_{i}$.

Since the height of the cotree is bounded by $k$, it follows that this algorithm can be implemented to run in $\mathrm{O}\left(\mathrm{k}^{\mathrm{k}^{2}} \cdot(\mathrm{k} \cdot|\mathrm{V}(\mathrm{G})|)^{5 / 2}\right)$. This proves the theorem.

\section{Foldings}

Definition 5. Let $\mathrm{G}=(\mathrm{V}, \mathrm{E})$ be a graph and let $\mathrm{x}$ and $\mathrm{y}$ be two vertices in $\mathrm{G}$ that are at distance two. A simple fold with respect to $\mathrm{x}$ and $\mathrm{y}$ is the operation which identifies $\mathrm{x}$ and $\mathrm{y}$. A folding is a homomorphisms which is a sequence of simple folds.

When $\mathrm{G} \rightarrow \mathrm{H}$ is a folding then we say that $\mathrm{G}$ folds onto $\mathrm{H}$.

It is well-known that any retraction is a folding, see eg [30, Proposition 2.19].

Definition 6. The folding number $\Sigma(\mathrm{G})$ of a connected graph $\mathrm{G}$ is the largest number s such that $\mathrm{G}$ folds onto $\mathrm{K}_{\mathrm{s}}$. When $\mathrm{G}$ is disconnected the folding number is the maximal folding number of the graphs induced by the components of $\mathrm{G}$.

Recall that the achromatic number $\Psi(\mathrm{G})$ of a graph $\mathrm{G}$ is the largest number of colors with which one can properly color the vertices of $G$ such that for any two colors there are two adjacent vertices that have those colors.

Lemma 6. Assume that $\mathrm{G}$ has a universal vertex $\mathrm{u}$. Then

$$
\Sigma(\mathrm{G})=1+\Psi(\mathrm{G}-\mathrm{u})=\Psi(\mathrm{G}) .
$$


Proof. Any two nonadjacent vertices of $\mathrm{G}-\mathrm{u}$ are at distance two in $\mathrm{G}$. Thus any achromatic coloring of $\mathrm{G}$ is a folding. The universal vertex must be in a color class by itself. Harary and Hedetniemi [31] show that, when $G$ is the join of two graphs $\mathrm{G}_{1}$ and $\mathrm{G}_{2}$ then $\Psi(\mathrm{G})=\Psi\left(\mathrm{G}_{1}\right)+\Psi\left(\mathrm{G}_{2}\right)$. The proves the lemma.

Notice that the achromatic number problem is NP-complete, even for trees. However, the problem is fixed-parameter tractable [15 43 46]. The image of a tree after a simple fold is a tree. Therefore, the folding number of a tree is at most two.

Theorem 8. The problem to compute the folding number is NP-complete, even when restricted to trivially perfect graphs.

Proof. Bodlaender shows in [3] that computing the achromatic number is NPcomplete, even when restricted to trivially perfect graphs. Since the class of trivially perfect graphs is closed under adding universal vertices, by Lemma 6 computing the folding number is NP-complete for trivially perfect graphs.

Theorem 9. When $\mathrm{G}$ is a threshold graph then

$$
\chi(\mathrm{G})=\Sigma(\mathrm{G})=\Psi(\mathrm{G}) .
$$

Proof. When $\mathrm{G}$ is the join of two graph $\mathrm{G}_{1}$ and $\mathrm{G}_{2}$ then

$$
\Psi(\mathrm{G})=\Psi\left(\mathrm{G}_{1}\right)+\Psi\left(\mathrm{G}_{2}\right) .
$$

Assume that $G$ has an isolated vertex $x$. In any achromatic coloring, the vertex must have a color that is used by another vertex also. Therefore,

$$
\Psi(G)=\max \{1, \Psi(G-x)\} .
$$

This proves the theorem.

\section{Absolute retracts for cographs}

Definition 7. Let $\mathcal{G}$ be a class of graphs. A graph $\mathrm{H}$ is an absolute retract for $\mathcal{G}$ if $\mathrm{H}$ is a retract of a graph $\mathrm{G} \in \mathcal{G}$ whenever $\mathrm{G}$ is an isometric embedding of $\mathrm{H}$ and $\chi(\mathrm{H})=\chi(\mathrm{G})$.

Hell, in his $\mathrm{PhD}$ thesis, characterized absolute retracts for the class of bipartite graphs as the retracts of components of categorical products of paths [33]. Pesch and Poguntke characterized absolute retracts of k-chromatic graphs [50]. Their characterization can be strengthened for the case of bipartite graphs such that it leads to a polynomial recognition algorithm for absolute retracts of bipartite graphs [2]. Examples of absolute retracts of bipartite graphs are the chordal bipartite graphs [24]. Median graphs are exactly the absolute retracts of hypercubes [1]. For reasons of brevity we leave out the mention of all results on reflexive graphs. 
To illustrate the problem for cographs, let $\mathrm{G}$ be the butterfly, ie $\mathrm{G}=\mathrm{K}_{1} \otimes\left(\mathrm{K}_{2} \oplus\right.$ $\mathrm{K}_{2}$ ), and let $\mathrm{H}$ be the paw, ie $\mathrm{H}=\mathrm{K}_{1} \otimes\left(\mathrm{K}_{1} \oplus \mathrm{K}_{2}\right)$. The $\mathrm{H}$ is an induced subgraph of $G$ and, obviously, $H$ has an isometric embedding in $G$ and $\omega(G)=\omega(H)$. But $\mathrm{H}$ is not a retract of $\mathrm{G}$ and so $\mathrm{H}$ is not an absolute retract for cographs.

Theorem 10. Let $\mathrm{H}$ be a connected cograph. Then $\mathrm{H}$ is an absolute retract for the class of cographs if and only if every vertex of $\mathrm{H}$ is in a maximal clique of cardinality $\omega(\mathrm{H})$.

Proof. First notice that a cograph $\mathrm{G}$ is an isometric embedding of a connected cograph $\mathrm{H}$ if and only if $\mathrm{H}$ is an induced subgraph of $\mathrm{G}$. This follows since connected cographs have diameter two or, also, because they are distance hereditary. The observation is true for distance-hereditary graphs simply by definition [36].

Let $\mathrm{H}$ be a connected cograph. Write $\omega=\omega(\mathrm{H})$ and assume that every vertex of $\mathrm{H}$ is in a clique of cardinality $\omega$. Let $\mathrm{G}$ be a cograph with $\omega(\mathrm{G})=\omega$ such that $\mathrm{H}$ is an induced subgraph of $\mathrm{G}$.

First assume that $\mathrm{G}$ is disconnected. Then the vertices of $\mathrm{H}$ are contained in one component of $\mathrm{G}$ since $\mathrm{H}$ is connected. If $\mathrm{W}$ is any other component, then $\mathrm{G}[\mathrm{W}]$ has clique number at most $\omega$ and so there is a homomorphism from this component to the component that contains $\mathrm{H}$. In other words, $\mathrm{H}$ is a retract of $\mathrm{G}$ if and only if $\mathrm{H}$ is a retract of the component that contains $\mathrm{H}$ as an induced subgraph. Henceforth, we may assume that $\mathrm{G}$ is connected.

Consider a cotree for $\mathrm{G}$. Since $\mathrm{G}$ is connected the root is an $\otimes$-node. Let

$$
\mathrm{C}_{1}, \ldots, \mathrm{C}_{\mathrm{t}}
$$

be the cocomponents of $G$. Since $H$ is an induced subgraph with the same cliquenumber as $\mathrm{G}, \mathrm{H}$ decomposes into the same number of cocomponents $\mathrm{D}_{1}, \ldots, \mathrm{D}_{\mathrm{t}}$. Notice that

$$
\omega=\sum_{i=1}^{t} \omega\left(G\left[C_{i}\right]\right)=\sum_{i=1}^{t} \omega\left(H\left[D_{i}\right]\right) .
$$

Therefore, since $\omega\left(H\left[D_{i}\right]\right) \leqslant \omega\left(G\left[C_{i}\right]\right)$, we have equality for each $i$, that is,

$$
\omega\left(H\left[D_{i}\right]\right)=\omega\left(G\left[C_{i}\right]\right) \quad \text { for } i \in\{1, \ldots, t\} .
$$

Now consider an $\oplus$-node. Let $C_{1}^{\prime}, \ldots, C_{\ell}^{\prime}$ be the sets of vertices of the subgraphs of $\mathrm{G}$ induced by the children. Let

$$
\omega^{\prime}=\max \left\{\omega\left(G\left[C_{i}^{\prime}\right]\right) \mid i \in\{1, \ldots, \ell\}\right\} .
$$

Write

$$
\mathrm{D}_{\mathrm{i}}^{\prime}=\mathrm{V}(\mathrm{H}) \cap \mathrm{C}_{\mathrm{i}}^{\prime} \quad \text { for } i \in\{1, \ldots, \ell\}
$$


Since $\omega(G)=\omega(H)$, we have that there is at least one component $C_{i}^{\prime}$ such that

$$
\omega^{\prime}=\omega\left(H\left[D_{i}^{\prime}\right]\right) .
$$

For $\mathrm{G}$ to retract to $\mathrm{H}$ we must have that, for every $j \in\{1, \ldots, \ell\}$,

$$
\mathrm{D}_{j}^{\prime} \neq \varnothing \quad \text { implies } \quad \omega\left(\mathrm{H}\left[\mathrm{D}_{\mathrm{j}}^{\prime}\right]\right)=\omega\left(\mathrm{G}\left[\mathrm{C}_{\mathrm{j}}^{\prime}\right]\right) \text {. }
$$

This condition is satisfied by virtue of the condition that every vertex of $\mathrm{H}$ is in a clique of cardinality $\omega$. Namely, this implies that for every $j \in\{1, \ldots, \ell\}$,

$$
\mathrm{D}_{\mathrm{j}}^{\prime} \neq \varnothing \quad \text { implies } \omega\left(\mathrm{H}\left[\mathrm{D}_{\mathrm{j}}^{\prime}\right]\right)=\omega^{\prime} .
$$

Notice that this condition is necessary for $\mathrm{H}$ to be an absolute retract. This can be seen as follows. If there were a component $D_{j}$ with

$$
\omega\left(H\left[D_{j}^{\prime}\right]\right)<\max \left\{\omega\left(H\left[D_{i}^{\prime}\right]\right) \mid i \in\{1, \ldots, \ell\}\right\}
$$

then we could construct a cograph $G$ such that $H$ is an induced subgraph of $G$ with $\omega(\mathrm{G})=\omega(\mathrm{H})$ but such that $\mathrm{G}$ does not contract to $\mathrm{H}$. Namely, add one vertex to a component $\mathrm{D}_{j}^{\prime}$ satisfying (5) as a true twin of a vertex which is in a maximum clique of $\mathrm{H}\left[\mathrm{D}_{j}^{\prime}\right]$.

This proves the theorem.

\section{Concluding remarks}

One interesting problem that we leave open is whether the folding number is fixed-parameter tractable.

We proved that the retract problem for cographs is fixed-parameter tractable when parameterized by the number of vertices in the smaller of the two graphs. Perhaps more challenging and useful would be the 'cleaning-parameter,' ie, the difference between the number of vertices of the two graphs, recently introduced by Marx and Schlotter [44/45]. This parameterization was investigated for the induced subgraph isomorphism problem, when restricted to various classes of graphs, eg, interval graphs, trees and planar graphs, and grids. As far as we know, whether cographs can be cleaned by a fixed-parameter algorithm is an open problem.

\section{References}

1. Bandelt, H., Retracts of hypercubes, Journal of Graph Theory 8 (1984), pp. 501-510.

2. Bandelt, H., A. Dählmann and H. Schütte, Absolute retracts of bipartite graphs, Discrete Applied Mathematics 16 (1987), pp. 191-215. 
3. Bodlaender, H., Achromatic number is NP-complete for cographs and interval graphs, Information Processing Letters 31 (1989), pp. 135-138.

4. Bulatov, A., The complexity of the counting constraint satisfaction problem, Proceedings of the $35^{\text {th }}$ International Colloquium on Automata, Languages and Programming, Springer-Verlag, LNCS 5125 (2008), pp. 646-661.

5. Bulatov, A., V. Dalmau, M. Grohe and D. Marx, Enumerating homomorphisms, Journal of Computer and System Sciences 78 (2012), pp. 638-650.

6. Courcelle, B. and S. Oum, Vertex-minors, monadic second-order logic, and a conjecture by Seese, Journal of Combinatorial Theory, Series B 97, (2007), pp. 91-126.

7. Chudnovsky, M., G. Cornuéjols, X. Liu, P. Seymour and K. Vušković, Recognizing Berge graphs, Combinatorica 25 (2005), pp. 143-186.

8. Chudnovsky, M., N. Robertson, P. Seymour and R. Thomas, The strong perfect graph theorem, Annals of Mathematics 164 (2006), pp. 51-229.

9. Chung, M., $\mathrm{O}\left(\mathrm{n}^{2.5}\right)$ time algorithms for the subgraph homomorphism problem on trees, Journal of Algorithms 8 (1987), pp. 106-112.

10. Corneil, D., H. Lerchs and L. Stewart-Burlingham, Completent reducible graphs, Discrete Applied Mathematics 3 (1981), pp. 163-174.

11. Corneil, D., Y. Perl and L. stewart, A linear recognition algorithm for cographs, SIAM Journal on Computing 14 (1985), pp. 926-934.

12. Damaschke, P., Induced subgraph isomorphism for cographs is NP-complete, Proceedings $16^{\text {th }} W G^{\prime} 90$, Springer-Verlag, LNCS 484 (1991), pp. 72-78.

13. Dyer, M. and D. Richerby, An effective dichotomy for the counting constraint satisfaction problem. Manuscript on ArXiv: 1003.3879, 2011.

14. Edmonds, J., Paths, trees, and flowers, Canadian Journal of Mathematics 17 (1965), pp. 449-467.

15. Farber, M., G. Hahn, P. Hell and D. Miller, Concerning the achromatic number of graphs, Journal of Combinatorial Theory, Series B 40 (1986), pp. 21-39.

16. Feder, T., P. Hell and J. Huang, List homomorphisms and circular arc graphs, Combinatorica 19 (1999), pp. 487-505.

17. Feder, T., P. Hell, P. Johnsson, A. Krokhin and G. Nordh, Retractions to pseudoforests, SIAM Journal on Discrete Mathematics 24 (2010), pp. 101-112.

18. Fomin, F., P. Heggernes and D. Kratsch, Exact algorithms for graph homomorphisms, Theory of Computing Systems 41 (2007), pp. 381-393.

19. Garey, M. and D. Johnson, Computers and intractability: a guide to the theory of NPcompleteness, W. H. Freeman and co., 1979.

20. Gioan, E. and C. Paul, Split-decomposition and graph-labelled trees: characterizations and fully dynamic algorithms for totally decomposable graphs. Manuscript on ArXiv: 0810.1823, 2008.

21. Goldberg, L., M. Grohe, M. Jerrum and M. Thurley, A complexity dichotomy for partition functions with mixed signs, SIAM Journal on Computing 39 (2010), pp. 33363402.

22. Golovach, P., B. Lidický, B. Martin and D. Paulusma, Finding vertex-surjective graph homomorphisms, Acta Informatica 49 (2012), pp. 381-394.

23. Golumbic, M., Trivially perfect graphs, Discrete Mathematics 24 (1978), pp. 105-107.

24. Golumbic, M. and C. Goss, Perfect elimination and chordal bipartite graphs, Journal of Graph Theory 2 (1978), pp. 155-163.

25. Götz, M., C. Koch and W. Martens, Efficient algorithms for the tree homeomorphism problem, Proceedings of the $11^{\text {th }}$ International Conference on Database Programming Languages, Springer-Verlag, LNCS 4797 (2007), pp. 17-31.

26. Grohe, M., The complexity of homomorphism and constraint satisfaction problems seen from the other side, Journal of the ACM, 54 (2007). 
27. Grohe, M., Personal communication.

28. Grohe, M., K. Kawarabayashi, D. Marx and P. Wollan, Finding topological subgraphs is fixed-parameter tractable, STOC'11, Proceedings of the $43^{\text {rd }}$ Annual ACM Symposium on Theory of Computing, ACM (2011), pp. 479-488.

29. Grötschel, M., L. Lovász and A. Schrijver, Polynomial algorithms for perfect graphs. In (Berge, Chvátal eds.): Topics on perfect graphs, North-Holland, Mathematics Studies 88 (1984), pp. 325-256.

30. Hahn, G. and C. Tardif, Graph homomorphisms: structure and symmetry. In (G. Hahn and G. Sabidussi eds.) Graph symmetry - algebraic methods and applications, NATO ASI Series C: Mathematical and Physical Sciences, Vol. 497, Kluwer, 1997, pp. 107166.

31. Harary, F. and S. Hedetniemi, The achromatic number of a graph, Journal of Combinatorial Theory 8 (1970), pp. 154-161.

32. Hell, P., Rétractions de graphes. PhD Thesis, Université de Montréal, 1972.

33. Hell, P. and J. Nešetřil, On the complexity of H-coloring, Journal of Combinatorial Theory, Series B 48 (1990), pp. 92-110.

34. Hell, P. and J. Nešetřil, The core of a graph, Discrete Mathematics 109 (1992), pp. 117-126.

35. Hell, P. and J. Nešetřil, Graphs and homomorphisms, Oxford Universty Press, 2004.

36. Howorka, E., A characterization of distance-hereditary graphs, The Quarterly Journal of Mathematics 28 (1977), pp. 417-420.

37. Imrich, W. and S. Klavžar, Retracts of strong products of graphs, Discrete Mathematics 109 (1992), pp. 147-154.

38. Kilpeläinen, P. and H. Mannila, Quey primitives for tree-structured data, Proceedings of the $5^{\text {th }}$ Annual Symposium on Combinatorial Pattern Matching, Springer-Verlag, LNCS 807 (1994), pp. 213-225.

39. Kilpeläinen, P. and H. Mannila, Ordered and unordered tree inclusion, SIAM Journal on Computing 24 (1995), pp. 340-356.

40. Klavžar, S., Absolute retracts of splitgraphs, Discrete Mathematics 134 (1994), pp. 75 84.

41. Kruskal, J., Well-quasi-ordering, the tree theorem, and Vazsonyi's conjecture, Transactions of the American Mathematical Society 95 (1960), pp. 210-225.

42. Loten, C., Retractions of chordal and related graphs. PhD Thesis, Simon Fraser University, 2003.

43. Manlove, D. and C. McDiarmid, The complexity of harmonious coloring for trees, Discrete Applied Mathematics 57 (1995), pp. 133-144.

44. Marx, D. and I. Schlotter, Parameterized graph cleaning problems, Discrete Applied Mathematics 157 (2009), pp. 3258-3267.

45. Marx, D. and I. Schlotter, Cleaning interval graphs, Algorithmica 65 (2013), pp. 275316.

46. Máté, A., A lower estimate for the achromatic number of irreducible graphs, Discrete Mathematics 33 (1981), pp. 171-183.

47. Matoušek and R. Thomas, On the complexity of finding iso- and other morphisms for partial k-trees, Discrete Mathematics 108 (1992), pp. 343-364.

48. Naserasr, R. and Y. Nigussie, On a new reformulation of Hadwiger's conjecture, Discrete Mathematics 306 (2006), pp. 3136-3139.

49. Nešetřil, J. and P. de Mendez, Cuts and bounds, Discrete Mathematics 302 (2005), pp. 211-224.

50. Pesch, E. and W. Poguntke, A characterization of absolute retracts of n-chromatic graphs, Discrete Mathematics 57 (1985), pp. 99-104. 
51. Pinter, R., O. Rokhlenko, D. Tsur and M. Ziv-Ukelson, Approximate labelled subtree homeomorphism, Journal of Discrete Algorithms 6 (2008), pp. 480-496.

52. Reyner, S., An analysis of good algorithms for the subtree problem, SIAM Journal on Computing 6 (1977), pp. 730-732.

53. Shamir, R. and D. Tsur, Faster subtree isomorphism, Journal of Algorithms 33 (1999), pp. 267-280.

54. Sikora, F., An (almost complete) state of the art around the graph motif problem. Manuscript 2012.

55. Thomassé, S., On better-quasi-ordering countable series-parallel orders, Transactions of the American Mathematical Society 352 (2000), pp. 2491-2505.

56. Vikas, N., A complete and equal computational complexity classification of compaction and retraction to all graphs with at most four vertices and some general results, Journal of Computer and System Sciences 71 (2005), pp. 406-439.

57. Wahlström, M., New plain-exponential time classes for graph homomorphism, Theory of Computing Systems 49 (2011), pp. 273-282.

58. Wolk, E., A note on "The comparability graph of a tree," Proceedings of the American Mathematical Society 16 (1965), pp. 17-20. 